\title{
Degenerate Sumudu Transform and Its Properties
}

\author{
Ugur Duran \\ Department of Basic Sciences of Engineering, \\ Faculty of Engineering and Natural Sciences, \\ Iskenderun Tecnical University, TR-31200 Hatay, Turkey \\ E-Mail: mtdrnugur@gmail.com
}

\begin{abstract}
Kim-Kim (Russ. J. Math. Phys. 2017, 24, 241-248) defined the degenerate Laplace transform and investigated some of their certain properties. Motivated by this study, in this paper, we introduce the degenerate Sumudu transform and establish some properties and relations. We derive degenerate Sumudu transforms of power functions, degenerate sine, degenerate cosine, degenerate hyperbolic sine, degenerate hyperbolic cosine, degenerate exponential function, and function derivatives. We also acquire a relationship between degenerate Sumudu transform and degenerate gamma function. Moreover, we investigate a scale preserving theorem for the degenerate Sumudu transform. Furthermore, we show that the degenerate Sumudu transform is the theoretical dual transform to the degenerate Laplace transform.
\end{abstract}

2010 Mathematics Subject Classification- Primary 44A10; Secondary 44A35, 44A30.

Keywords and Phrases- Degenerate exponential function; degenerate gamma function; Sumudu transform; Laplace transform.

\section{INTRODUCTION}

The integral transforms play a key role in solving solutions to initial value problems and initial boundary value problems. The integral transform was considered by the French physicists and mathematician P.S. Laplace [17] in 1780, which has very powerful applications, not only in applied mathematics but also in other branches of science such as engineering, physics, astronomy, etc. From Laplace time to now, several integral transforms such as Fourier, Sumudu, Elzaki, and $M$-transforms have been defined, and some of their properties and applications have been studied in detail, $c f .[1-4,7,11-13,16-21]$ and see also each of the references cited therein.

In 1993, Watugula [19] defined the Sumudu transform, which is the theoretical dual to the Laplace transform, and gave many interesting properties. Then, many mathematicians and physicists have studied Sumudu transform and investigated diverse applications of this transform, cf. [1-4,17,19-21]. For instance, Watagula [20] considered the Sumudu transform for functions of two variables and gave an application solving partial differential equations with known initial conditions. Weerakoon [21] derived the Sumudu transform of partial derivatives and showed its applicability demonstrated using three different partial differential equations. Asiru [1] gave the Sumudu transform of some special functions and provided some examples with Abel's integral equation, an integro-differential equation, a dynamic system with delayed time signals, and a differential dynamic system. Belgacem et al. [2] provided fundamental properties including scale and unit-preserving properties of Sumudu transform and gave a solution an integral production-depreciation problem. Belgacem [3] examined deeper Sumudu properties and connections. Belgacem et al. [4] generalize all existing Sumudu differentiation, integration, and convolution theorems and Sumudu shifting theorems. In this study, we define degenerate Sumudu transform and provide some of their properties and relations. We acquire degenerate Sumudu transforms of power functions, degenerate sine, degenerate cosine, degenerate hyperbolic sine, degenerate hyperbolic cosine, degenerate exponential function, and function derivatives. We also attain a relation between degenerate Sumudu transform and degenerate gamma function. Moreover, we derive a scale preserving theorem for the degenerate Sumudu transform. Lastly, we give duality between degenerate Laplace transform and degenerate Sumudu transform. 
The gamma function is defined by the following improper integral (cf. [1-4,7,11-13,16-21]):

$$
\Gamma(s)=\int_{0}^{\infty} e^{-t} t^{s-1} d t,
$$

where $s$ being a complex number with $\operatorname{Re}(s)>0$. The gamma function satisfies the following relations

$$
\Gamma(s+1)=s \Gamma(s) \text { and } \Gamma(n+1)=n \text { ! }
$$

for $n$ being a non-negative integer.

Let $f$ be a function defined for $t \geq 0$. Then the following integral

$$
F(s)=\mathcal{L}(f(t))=\int_{0}^{\infty} e^{-s t} f(t) d t
$$

is said to be the Laplace transform of $f$, provided that the integral converges, $c f$. $[2-4,7,11,12,16-18]$.

\section{Preliminaries and Definitions}

Here are some basic notations and definitions which will be used in the next sections.

Throughout this paper, the familiar symbols $\mathbb{C}, \mathbb{R}, \mathbb{Z}, \mathbb{N}$ and $\mathbb{N}_{0}$ are referred to as the set of all complex numbers, the set of all real numbers, the set of all integers, the set of all natural numbers, and the set of all non-negative integers, respectively.

For $r \in \mathbb{C}$, the $r$-falling factorial $(x)_{n, r}$ is defined by (see [5,6,8-15])

$$
(x)_{n, r}= \begin{cases}x(x-r)(x-2 r) \cdots(x-(n-1) r), & n=1,2, \ldots \\ 1 & n=0 .\end{cases}
$$

In the case $r=1$, the $r$-falling factorial reduces to the familiar falling factorial (see [5,6,8-15])

$$
(x)_{n, 1}:=(x)_{n}=x(x-1) \cdots(x-n+1) .
$$

The degenerate exponential function $e_{\lambda}^{x}(t)$ for a real number $\lambda$ is given by $(c f$. [5,6,8-15])

$$
e_{\lambda}^{x}(t)=(1+\lambda t)^{\frac{x}{\lambda}} \text { and } e_{\lambda}^{1}(t)=e_{\lambda}(t) .
$$

It is readily seen that $\lim _{\lambda \rightarrow 0} e_{\lambda}^{x}(t)=e^{x t}$. From (2.1) and (2.2), we obtain the derivative of the degenerate exponential function:

$$
\frac{d}{s x} e_{\lambda}^{x}(t)=\frac{1}{\lambda} \log (1+\lambda t) e_{\lambda}^{x}(t) .
$$

For $\lambda \in(0, \infty)$, the degenerate gamma function is defined by Kim-Kim [11] for the complex variable $s$ with $0<\operatorname{Re}(s)<\frac{1}{\lambda}$ by the following improper integral:

$$
\Gamma_{\lambda}(s)=\int_{0}^{\infty} e_{\lambda}^{-1}(t) t^{s-1} d t
$$

and the degenerate Laplace transform is also defined by Kim-Kim [11] as follows:

$$
\mathcal{L}_{\lambda}(f(t))=F_{\lambda}(s)=\int_{0}^{\infty} e_{\lambda}^{-s}(t) f(t) d t,
$$

if the integral converges. Several properties and interesting formulas for the degenerate gamma function and degenerate Laplace transform are derived in [11]. Then, Kim et al. [12] defined modified degenerate gamma function and modified degenerate Laplace transform, and investigate various formulas and basic properties. Kim-Kim [13] derived diverse properties of the degenerate gamma function, including an analytic continuation as a meromorphic function on the whole complex plane, the values at positive integers, the difference formula, several expressions following from the Euler and Weierstrass formulae for the ordinary gamma function, and an integral representation as an integral along a Hankel contour.

Note that

$$
\lim _{\lambda \rightarrow 0} \Gamma_{\lambda}(s)=\Gamma(s) \text { and } \lim _{\lambda \rightarrow 0} \mathcal{L}_{\lambda}(f(t))=\mathcal{L}(f(t)) .
$$




\section{Degenerate Sumudu Transform}

In this chapter, we consider the degenerate Sumudu transform and investigate some of their properties. Over the set of functions

$$
A=\left\{f(t)\left|\exists M, \tau_{1}, \tau_{2}>0,\right| f(t) \mid<M e^{\frac{|t|}{\tau_{j}}}, \text { if } t \in(-1)^{j} \times[0, \infty)\right\}
$$

the Sumudu transform is defined by the following improper integral:

$$
G(u)=\mathbf{S}[f(t)]=\int_{0}^{\infty} e^{-t} f(u t) d t=\frac{1}{u} \int_{0}^{\infty} e^{-\frac{t}{u}} f(t) d t, \quad u \in\left(-\tau_{1}, \tau_{2}\right) .
$$

The Sumudu transform is usually used to solve ordinary differential equations and engineering control problems, $c f$. $[1-4,17,19-21]$ and see the references cited therein.

The Sumudu transformation satisfies the following operational properties, $c f .[2,4]$ :

\begin{tabular}{|c|c|}
\hline $\mathbf{S}[1]=1$ & $\mathbf{S}[\sin (a t)]=\frac{a u}{1+u^{2} a^{2}}$ \\
\hline $\mathbf{S}[t]=u$ & $\mathbf{S}[\cos (a t)]=\frac{1}{1+u^{2} a^{2}}$ \\
\hline $\mathbf{S}\left[t^{n}\right]=n ! u^{n}$ & $\mathbf{S}[\sinh (a t)]=\frac{a u}{1+u^{2} a^{2}}$ \\
\hline $\mathbf{S}\left[e^{a t}\right]=\frac{1}{1-u a}$ & $\mathbf{S}[\cosh (a t)]=\frac{1}{1+u^{2} a^{2}}$ \\
\hline $\mathbf{S}[f(a t)]=G(a u)$ & $\mathbf{S}\left[e^{a t} f(t)\right]=\frac{1}{1-a u} G\left(\frac{u}{1-a u}\right)$ \\
\hline
\end{tabular}

Let $f(t), g(t) \in A$ be Sumudu transforms $M(u)$ and $N(u)$, respectively. Then the Sumudu transform of the convolution of $f$ and $g$ is given by

$$
\mathbf{S}[(f * g)(t)]=u M(u) N(u),
$$

where the convolution integral is given by $(c f .[2,4])$

$$
(f * g)(t)=\int_{0}^{t} g(x) f(t-x) d x
$$

Now, we give our main definition.

Definition 1. Let $\lambda \in(0, \infty)$ and

$$
A_{\lambda}=\left\{f(t) \mid \exists M, \tau_{1}, \tau_{2}>0 \text { such that }|f(t)|<M e_{\lambda}^{1 / \tau_{j}}(|t|), \text { if } t \in(-1)^{j} \times[0, \infty)\right\} .
$$

Then for $f(t) \in A_{\lambda}$, we define the degenerate Sumudu transform is defined by the following improper integral:

$$
G_{\lambda}(u)=\mathbf{S}_{\lambda}[f(t)]=\frac{1}{u} \int_{0}^{\infty} e_{\lambda}^{\frac{-1}{u}}(t) f(t) d t, \quad u \in\left(-\tau_{1}, \tau_{2}\right)
$$

We note that

$$
\lim _{\lambda \rightarrow 0} \mathbf{S}_{\lambda}[f(t)]=\mathbf{S}[f(t)] .
$$

Let $f(t), g(t) \in A_{\lambda}$ and $\alpha, \beta \in \mathbb{R}$. The degenerate Sumudu transformation is a linear transform, namely

$$
\begin{aligned}
\mathbf{S}_{\lambda}[\alpha f(t)+\beta g(t)] & =\frac{1}{u} \int_{0}^{\infty} e_{\lambda}^{\frac{-1}{u}}(t)[\alpha f(t)+\beta g(t)] d t \\
& =\frac{\alpha}{u} \int_{0}^{\infty} e_{\lambda}^{\frac{-1}{u}}(t) f(t) d t+\frac{\beta}{u} \int_{0}^{\infty} e_{\lambda}^{\frac{-1}{u}}(t) g(t) d t \\
& =\alpha \mathbf{S}_{\lambda}[f(t)]+\beta \mathbf{S}_{\lambda}[g(t)] .
\end{aligned}
$$


By Definition 1 , for $f(t)=1$, we observe that

$$
\begin{aligned}
\mathbf{S}_{\lambda}[1] & =\frac{1}{u} \int_{0}^{\infty} e_{\lambda}^{\frac{-1}{u}}(t) d t=\lim _{R \rightarrow \infty} \frac{1}{u} \int_{0}^{R}(1+\lambda t)^{-\frac{1}{\lambda u}} d t \\
& =\left.\frac{1}{\lambda u} \lim _{R \rightarrow \infty} \frac{(1+\lambda t)^{1-\frac{1}{u \lambda}}}{1-\frac{1}{u \lambda}}\right|_{0} ^{R}=\lim _{R \rightarrow \infty}\left(\frac{(1+\lambda R)^{\frac{u \lambda-1}{u \lambda}}}{\lambda u-1}-\frac{1}{\lambda u-1}\right) \\
& =\frac{1}{1-\lambda u}, \quad u \lambda<1,
\end{aligned}
$$

and for $f(t)=t$ with $2 u \lambda<1$,

$$
\begin{aligned}
\mathbf{S}_{\lambda}[t] & =\frac{1}{u} \int_{0}^{\infty} t e_{\lambda}^{\frac{-1}{u}}(t) d t=\lim _{R \rightarrow \infty}\left(\left.t \frac{(1+\lambda t)^{\frac{\lambda u-1}{\lambda u}}}{\lambda u-1}\right|_{0} ^{R}-\frac{1}{\lambda u-1} \int_{0}^{R}(1+\lambda t)^{1-\frac{1}{\lambda u}}\right) d t \\
& =\left.\lim _{R \rightarrow \infty} \frac{u}{u \lambda-1} \frac{(1+\lambda t)^{2-\frac{1}{\lambda u}}}{2 \lambda u-1}\right|_{0} ^{R} d t \\
& =\lim _{R \rightarrow \infty} \frac{u}{1-\lambda u}\left(\frac{(1+\lambda R)^{2-\frac{1}{\lambda u}}}{2 \lambda u-1}-\frac{1}{2 \lambda u-1}\right) \\
& =\frac{u}{(1-\lambda u)(1-2 \lambda u)}
\end{aligned}
$$

Therefore, we state the following results.

Theorem 1. Each of the following degenerate Sumudu transforms is valid:

$$
\mathbf{S}_{\lambda}[1]=\frac{1}{1-\lambda u}, \quad \text { for } \lambda u<1
$$

and

$$
\mathbf{S}_{\lambda}[t]=\frac{u}{(1-\lambda u)(1-2 \lambda u)}, \text { for } 2 \lambda u<1 .
$$

Remark 1. It is clear from Theorem 1 that

$$
\lim _{\lambda \rightarrow 0} \mathbf{S}_{\lambda}[1]=\lim _{\lambda \rightarrow 0} \frac{1}{1-\lambda u}=1=\mathbf{S}[1]
$$

and

$$
\lim _{\lambda \rightarrow 0} \mathbf{S}_{\lambda}[t]=\lim _{\lambda \rightarrow 0} \frac{u}{(1-\lambda u)(1-2 \lambda u)}=u=\mathbf{S}[t] .
$$

By Definition 1, for $f(t)=t^{n}$ with $n \in \mathbb{N}$ and $(n+1) u \lambda<1$, we observe that

$$
\begin{aligned}
\mathbf{S}_{\lambda}\left[t^{n}\right] & =\frac{1}{u} \int_{0}^{\infty} t^{n} e_{\lambda}^{\frac{-1}{u}}(t) d t=\frac{n}{1-\lambda u} \int_{0}^{\infty} t^{n-1} e_{\lambda}^{1-\frac{1}{u}}(t) d t \\
& =\frac{n}{1-\lambda u} \int_{0}^{\infty} t^{n-1} e_{\lambda}^{1-\frac{1}{u}}(t) d t \\
& =\frac{n(n-1)}{(1-\lambda u)(1-2 \lambda u)} u \int_{0}^{\infty} t^{n-2} e_{\lambda}^{2-\frac{1}{u}}(t) d t=\ldots \\
& =\frac{n ! u^{n-1}}{(1-\lambda u)(1-2 \lambda u) \cdots(1-n \lambda u)} \int_{0}^{\infty} e_{\lambda}^{n-\frac{1}{u}}(t) d t=\ldots \\
& =\frac{n ! u^{n}}{(1-\lambda u)(1-2 \lambda u) \cdots(1-(n+1) \lambda u)},
\end{aligned}
$$


and for $f(t)=e_{\lambda}^{a}(t)$,

$$
\begin{aligned}
\mathbf{S}_{\lambda}\left[e_{\lambda}^{a}(t)\right] & =\frac{1}{u} \int_{0}^{\infty} e_{\lambda}^{\frac{a}{\lambda}-\frac{1}{u}}(t) d t=\left.\lim _{R \rightarrow \infty} \frac{(1+\lambda t)^{\frac{a u-1}{\lambda u}+1}}{a u-1+u \lambda}\right|_{0} ^{R} \\
& =\lim _{R \rightarrow \infty}\left(\frac{(1+\lambda R)^{\frac{a u-1}{\lambda u}+1}}{u(a+\lambda)-1}-\frac{1}{u(a+\lambda)-1}\right) \\
& =\frac{1}{1-u(a+\lambda)}, \quad \frac{1-a u}{u \lambda}>1 .
\end{aligned}
$$

Thereby, we provide the degenerate Sumudu transforms of the power function and degenerate exponential function as follows.

Theorem 2. Let $n \in \mathbb{N}$. Each of the following degenerate Sumudu transforms is valid:

$$
\mathbf{S}_{\lambda}\left[t^{n}\right]=\frac{n ! u^{n}}{(1-\lambda u)(1-2 \lambda u) \cdots(1-(n+1) \lambda u)}, \text { for } \quad(n+1) u \lambda<1
$$

and

$$
\mathbf{S}_{\lambda}\left[e_{\lambda}^{a}(t)\right]=\frac{1}{1-u(a+\lambda)}, \text { for } u(a+\lambda)<1 .
$$

Remark 2. It is clear from Theorem 2 that

$$
\lim _{\lambda \rightarrow 0} \mathbf{S}_{\lambda}\left[t^{n}\right]=\lim _{\lambda \rightarrow 0} \frac{n ! u^{n}}{(1-\lambda u)(1-2 \lambda u) \cdots(1-(n+1) \lambda u)}=n ! u^{n}=\mathbf{S}\left[t^{n}\right]
$$

and

$$
\lim _{\lambda \rightarrow 0} \mathbf{S}_{\lambda}\left[e_{\lambda}^{a}(t)\right]=\lim _{\lambda \rightarrow 0} \frac{1}{1-u(a+\lambda)}=\frac{1}{1-u a}=\mathbf{S}\left[e^{a t}\right] .
$$

In [11], Kim et al. defined the degenerate $\operatorname{sine} \sin _{\lambda} t$ and $\operatorname{cosine} \cos _{\lambda} t$ functions by

$$
\sin _{\lambda}^{(x)}(t)=\frac{e_{\lambda}^{i x}(t)-e_{\lambda}^{-i x}(t)}{2 i} \text { and } \cos _{\lambda}^{(x)}(t)=\frac{e_{\lambda}^{i x}(t)+e_{\lambda}^{-i x}(t)}{2},
$$

where $i=\sqrt{-1}$. Note that $\lim _{\lambda \rightarrow 0} \sin _{\lambda}^{(x)}(t)=\sin x t$ and $\lim _{\lambda \rightarrow 0} \cos { }_{\lambda}^{(x)}(t)=\cos x t$. From (2.2) and (3.11), it is readily that

$$
e_{\lambda}^{i x}(t)=\cos _{\lambda}^{(x)}(t)+i \sin _{\lambda}^{(x)}(t) .
$$

From Definition 1 and using formulae (3.8) and (3.11), we have

$$
\begin{aligned}
\mathbf{S}_{\lambda}\left[\sin _{\lambda}^{(a)}(t)\right] & =\mathbf{S}_{\lambda}\left[\frac{e_{\lambda}^{i a}(t)-e_{\lambda}^{-i a}(t)}{2 i}\right] \\
& =\frac{1}{2 i}\left(\mathbf{S}_{\lambda}\left[e_{\lambda}^{i a}(t)\right]-\mathbf{S}_{\lambda}\left[e_{\lambda}^{-i a}(t)\right]\right) \\
& =\frac{1}{2 i}\left(\frac{1}{1-u(a i+\lambda)}-\frac{1}{1-u(-a i+\lambda)}\right) \\
& =\frac{a u}{(1-\lambda u)^{2}+u^{2} a^{2}}
\end{aligned}
$$


and

$$
\begin{aligned}
\mathbf{S}_{\lambda}\left[\cos _{\lambda}^{(a)}(t)\right] & =\mathbf{S}_{\lambda}\left[\frac{e_{\lambda}^{i a}(t)+e_{\lambda}^{-i a}(t)}{2}\right] \\
& =\frac{1}{2}\left(\mathbf{S}_{\lambda}\left[e_{\lambda}^{i a}(t)\right]+\mathbf{S}_{\lambda}\left[e_{\lambda}^{-i a}(t)\right]\right) \\
& =\frac{1}{2}\left(\frac{1}{1-u(a i+\lambda)}+\frac{1}{1-u(-a i+\lambda)}\right) \\
& =\frac{1-u \lambda}{(1-\lambda u)^{2}+u^{2} a^{2}} .
\end{aligned}
$$

Thus, we attain the degenerate Sumudu transforms of the degenerate sine function and degenerate cosine function as follows.

Theorem 3. Each of the following degenerate Sumudu transforms holds

$$
\mathbf{S}_{\lambda}\left[\sin _{\lambda}^{(a)}(t)\right]=\frac{a u}{(1-\lambda u)^{2}+u^{2} a^{2}},
$$

and

$$
\mathbf{S}_{\lambda}\left[\cos _{\lambda}^{(a)}(t)\right]=\frac{1-u \lambda}{(1-\lambda u)^{2}+u^{2} a^{2}} .
$$

Remark 3. It is obvious from Theorem 3 that

$$
\lim _{\lambda \rightarrow 0} \mathbf{S}_{\lambda}\left[\sin _{\lambda}^{(a)}(t)\right]=\lim _{\lambda \rightarrow 0} \frac{a u}{(1-\lambda u)^{2}+u^{2} a^{2}}=\frac{a u}{1+u^{2} a^{2}}=\mathbf{S}[\sin (a t)] .
$$

and

$$
\lim _{\lambda \rightarrow 0} \mathbf{S}_{\lambda}\left[\cos _{\lambda}^{(a)}(t)\right]=\lim _{\lambda \rightarrow 0} \frac{1-u \lambda}{(1-\lambda u)^{2}+u^{2} a^{2}}=\frac{1}{1+u^{2} a^{2}}=\mathbf{S}[\cos (a t)] .
$$

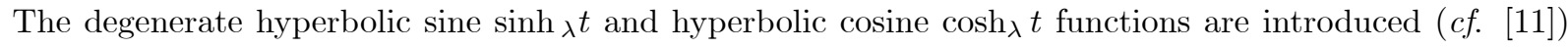
by

$$
\sinh _{\lambda}^{(x)}(t)=\frac{e_{\lambda}^{x}(t)-e_{\lambda}^{-x}(t)}{2} \text { and } \cosh _{\lambda}^{(x)}(t)=\frac{e_{\lambda}^{x}(t)+e_{\lambda}^{-x}(t)}{2} .
$$

Note that $\lim _{\lambda \rightarrow 0} \sinh _{\lambda}^{(x)}(t)=\sinh x t$ and $\lim _{\lambda \rightarrow 0} \cosh _{\lambda}^{(x)}(t)=\cosh x t$.

By Definition 1, and utilizing formulae (3.8) and (3.15), we derive

$$
\begin{aligned}
\mathbf{S}_{\lambda}\left[\sinh _{\lambda}^{(a)}(t)\right] & =\mathbf{S}_{\lambda}\left[\frac{e_{\lambda}^{a}(t)-e_{\lambda}^{-a}(t)}{2}\right] \\
& =\frac{1}{2}\left(\frac{1}{1-u(a+\lambda)}-\frac{1}{1-u(-a+\lambda)}\right) \\
& =\frac{a u}{(1-\lambda u)^{2}-u^{2} a^{2}}
\end{aligned}
$$

and

$$
\begin{aligned}
\mathbf{S}_{\lambda}\left[\cosh _{\lambda}^{(a)}(t)\right] & =\mathbf{S}_{\lambda}\left[\frac{e_{\lambda}^{a}(t)+e_{\lambda}^{-a}(t)}{2}\right] \\
& =\frac{1}{2}\left(\frac{1}{1-u(a+\lambda)}+\frac{1}{1-u(-a+\lambda)}\right) \\
& =\frac{1-u \lambda}{(1-\lambda u)^{2}-u^{2} a^{2}} .
\end{aligned}
$$

Therefore, we acquire the degenerate Sumudu transforms of the degenerate hyperbolic sine function and degenerate hyperbolic cosine function as follows. 
Theorem 4. Each of the following degenerate Sumudu transforms is valid:

$$
\mathbf{S}_{\lambda}\left[\sinh _{\lambda}^{(a)}(t)\right]=\frac{a u}{(1-\lambda u)^{2}-u^{2} a^{2}},
$$

and

$$
\mathbf{S}_{\lambda}\left[\cosh _{\lambda}^{(a)}(t)\right]=\frac{1-u \lambda}{(1-\lambda u)^{2}-u^{2} a^{2}} .
$$

Remark 4. It is obvious from Theorem 3 that

$$
\lim _{\lambda \rightarrow 0} \mathbf{S}_{\lambda}\left[\sinh _{\lambda}^{(a)}(t)\right]=\lim _{\lambda \rightarrow 0} \frac{a u}{(1-\lambda u)^{2}+u^{2} a^{2}}=\frac{a u}{1+u^{2} a^{2}}=\mathbf{S}[\sinh (a t)] .
$$

and

$$
\lim _{\lambda \rightarrow 0} \mathbf{S}_{\lambda}\left[\cosh _{\lambda}^{(a)}(t)\right]=\lim _{\lambda \rightarrow 0} \frac{1-u \lambda}{(1-\lambda u)^{2}+u^{2} a^{2}}=\frac{1}{1+u^{2} a^{2}}=\mathbf{S}[\cosh (a t)] .
$$

By Definition 1 and (2.4), for $\alpha \in \mathbb{R}$ with $\alpha>-1$ and $(\alpha+1) u \lambda<1$, we derive

$$
\begin{aligned}
\mathbf{S}_{\lambda}\left[t^{\alpha}\right] & =\frac{1}{u} \int_{0}^{\infty} t^{\alpha} e_{\lambda}^{\frac{-1}{u}}(t) d t=u^{\alpha-1} \int_{0}^{\infty}\left(\frac{t}{u}\right)^{\alpha} e_{\lambda u}^{\frac{-1}{u}}(t / u) d t \\
& =u^{\alpha} \int_{0}^{\infty} t^{\alpha} e_{\lambda u}^{-1}(t) d t=u^{\alpha} \Gamma_{\lambda u}(\alpha+1) .
\end{aligned}
$$

Thus, we arrive at the following relation between degenerate Sumudu transform and degenerate gamma function as follows.

Theorem 5. The following

$$
\mathbf{S}_{\lambda}\left[t^{\alpha}\right]=u^{\alpha} \Gamma_{\lambda u}(\alpha+1)
$$

is valid for $\alpha \in \mathbb{R}$ with $\alpha>-1$ and $(\alpha+1) u \lambda<1$.

We now investigate some formulas for degenerate Sumudu transform of function derivatives.

By Definition 1, we see that

$$
\mathbf{S}_{\lambda}\left[f^{(1)}(t)\right]=\frac{1}{u} \int_{0}^{\infty} f^{(1)}(t) e_{\lambda}^{\frac{-1}{u}}(t) d t=\frac{1}{u}\left(-f(0)+\mathbf{S}_{\lambda}\left[f(t) e_{\lambda}^{-1}(t)\right]\right) .
$$

By means of (3.16), we acquire

$$
\begin{aligned}
\mathbf{S}_{\lambda}\left[f^{(2)}(t)\right] & =\frac{1}{u} \int_{0}^{\infty} f^{(2)}(t) e_{\lambda}^{\frac{-1}{u}}(t) d t \\
& =\frac{1}{u^{2}}\left(-f(0)-u f^{(1)}(0)+(1+u \lambda) \mathbf{S}_{\lambda}\left[f(t) e_{\lambda}^{-2}(t)\right]\right) .
\end{aligned}
$$

Continuing this process, we get

$$
\begin{aligned}
\mathbf{S}_{\lambda}\left[f^{(n)}(t)\right]= & \frac{-1}{u^{n}} \sum_{i=0}^{n-1} u^{i} f^{(i)}(0) \prod_{l=1}^{n-i-2}(1+l u \lambda) \\
& +\frac{1}{u^{n}} \mathbf{S}_{\lambda}\left[f(t) e_{\lambda}^{-n}(t)\right] \prod_{l=1}^{n-1}(1+l u \lambda) .
\end{aligned}
$$

By (3.17), degenerate Sumudu transform of function derivatives is given below.

Theorem 6. The following degenerate Sumudu transform

$$
\mathbf{S}_{\lambda}\left[f^{(n)}(t)\right]=\frac{-1}{u^{n}} \sum_{i=0}^{n-1} u^{i} f^{(i)}(0) \prod_{l=1}^{n-i-2}(1+l u \lambda)+\frac{\mathbf{S}_{\lambda}\left[f(t) e_{\lambda}^{-n}(t)\right]}{u^{n}} \prod_{l=1}^{n-1}(1+l u \lambda) .
$$

is valid for $n \in \mathbb{N}$. 
By Definition 1 , for $u \in\left(-\tau_{1}, \tau_{2}\right)$, we observe that

$$
\begin{aligned}
\frac{d}{d u} G_{\lambda}(u) & =-\frac{1}{u^{2}} \int_{0}^{\infty} e_{\lambda}^{\frac{-1}{u}}(t) f(t) d t+\frac{1}{\lambda u^{3}} \int_{0}^{\infty} e_{\lambda}^{\frac{-1}{u}}(t) \log (1+\lambda t) f(t) d t \\
& =-\frac{1}{u} G_{\lambda}(u)+\frac{1}{\lambda u^{2}} \mathbf{S}_{\lambda}[f(t) \log (1+\lambda t)]
\end{aligned}
$$

and

$$
\begin{aligned}
\frac{d^{2}}{d u^{2}} G_{\lambda}(u)= & \frac{d}{d u}\left(-\frac{1}{u} G_{\lambda}(u)+\frac{1}{\lambda u^{2}} \mathbf{S}_{\lambda}[f(t) \log (1+\lambda t)]\right) \\
= & \frac{1}{u^{2}} G_{\lambda}(u)-\frac{1}{u}\left(-\frac{1}{u} G_{\lambda}(u)+\frac{1}{\lambda u^{2}} \mathbf{S}_{\lambda}[f(t) \log (1+\lambda t)]\right) \\
& -\frac{2}{\lambda u^{3}} \mathbf{S}_{\lambda}[f(t) \log (1+\lambda t)] \\
& +\frac{1}{\lambda u^{2}}\left(-\frac{1}{u} \mathbf{S}_{\lambda}[f(t) \log (1+\lambda t)]+\frac{1}{\lambda u^{2}} \mathbf{S}_{\lambda}\left[f(t)(\log (1+\lambda t))^{2}\right]\right) \\
= & \frac{2}{u^{2}} G_{\lambda}(u)-\frac{4}{\lambda u^{3}} \mathbf{S}_{\lambda}[f(t) \log (1+\lambda t)]+\frac{1}{\lambda^{2} u^{4}} \mathbf{S}_{\lambda}\left[f(t)(\log (1+\lambda t))^{2}\right] .
\end{aligned}
$$

Continuing this process, we arrive at the following result.

Theorem 7. Let $n \in \mathbb{N}$. The following derivative property is valid:

$$
\begin{gathered}
\frac{d^{n}}{d u^{n}} G_{\lambda}(u)=\sum_{k=0}^{n} a_{n, k} \frac{(-1)^{n-k}}{\lambda^{k} u^{n+k}}\left[f(t)(\log (1+\lambda t))^{k}\right] \\
(-1)^{n} \frac{n !}{u^{n}} G_{\lambda}(u)+(-1)^{n+1} \frac{n ! n}{\lambda u^{n+1}}+\cdots \\
-\frac{n^{2}}{\lambda^{n-1} u^{2 n-1}} \mathbf{S}_{\lambda}\left[f(t)(\log (1+\lambda t))^{n-1}\right]+\frac{1}{\lambda^{n} u^{2 n}}\left[f(t)(\log (1+\lambda t))^{n}\right]
\end{gathered}
$$

where $a_{n, 0}=n !, a_{n, 1}=n ! n, a_{n, n-1}=n^{2}, a_{n, n}=1$ and for $k=2,3, \ldots, n-2$,

$$
a_{n, k}=a_{n-1, k-1}+(n+k) a_{n-1, k} .
$$

From Definition 1, we observe that

$$
\mathbf{S}_{\lambda}[f(a t)]=\frac{1}{u} \int_{0}^{\infty} f(a t) e_{\lambda}^{\frac{-1}{u}}(t) d t=\frac{1}{u} \int_{0}^{\infty} f(a t) e_{\lambda / a}^{\frac{-1}{a u}}(a t) d t
$$

and upon setting $\omega=a t$, then

$$
\mathbf{S}_{\lambda}[f(a t)]=\frac{1}{a u} \int_{0}^{\infty} f(\omega) e_{\lambda / a}^{\frac{-1}{a u}}(\omega) d t=G_{\lambda / a}(a u) .
$$

Thereby, a scale preserving theorem for the degenerate Sumudu transform is given below.

Theorem 8. The following

$$
\mathbf{S}_{\lambda}[f(a t)]=G_{\lambda / a}(a u)
$$

holds.

Remark 5. It is obvious from Theorem 8 that

$$
\lim _{\lambda \rightarrow 0} \mathbf{S}_{\lambda}[f(a t)]=\lim _{\lambda \rightarrow 0} G_{\lambda / a}(a u)=G(a u)=\mathbf{S}[f(a t)] .
$$




\section{FURTher REMARKS}

By (1.1) and (3.1), the Sumudu transform is the theoretical dual transform to the Laplace transform given below $(c f .[2,4,19,20])$

$$
G(u)=\frac{F(1 / u)}{u} \text { and } F(s)=\frac{G(1 / s)}{s} .
$$

Using (2.5) and (3.6), for $f(t) \in A_{\lambda}$ and $-\tau_{1}<u<\tau_{2}$, we observe that

$$
G_{\lambda}(u)=\mathbf{S}_{\lambda}[f(t)]=\frac{1}{u} \int_{0}^{\infty} e_{\lambda}^{-\frac{1}{u}}(t) f(t) d t=\frac{1}{u} F_{\lambda}\left(\frac{1}{u}\right)
$$

and

$$
F_{\lambda}(s)=\mathcal{L}_{\lambda}(f(t))=\frac{1}{s} \frac{1}{\frac{1}{s}} \int_{0}^{\infty} e_{\lambda}^{-s}(t) f(t) d t=\frac{1}{s} G_{\lambda}\left(\frac{1}{s}\right)
$$

which are the degenerate version of the duality in (4.1). Therefore, the relations (4.2) and (4.3) between the degenerate Sumudu transform and the degenerate Laplace transform means to acquire one from the other when needed. For example, since $\mathcal{L}_{\lambda}\left(\cosh _{\lambda}(a t)\right)=\frac{s-\lambda}{(s-\lambda)^{2}-a^{2}}$, recall from Theorem 4, we have

$$
F_{\lambda}(s)=\frac{1}{s} \frac{-(1 / s) \lambda}{\left(1-\frac{1}{s} \lambda\right)^{2}-\left(\frac{1}{s}\right)^{2} a^{2}}=\frac{1}{s} G_{\lambda}\left(\frac{1}{s}\right) .
$$

Hence, we can say from (4.2) and (4.3) that the degenerate Sumudu transform is the theoretical dual transform to the degenerate Laplace transform.

\section{Conclusions}

Kim-Kim [11] defined the degenerate Laplace transform as follows:

$$
\mathcal{L}_{\lambda}(f(t))=F_{\lambda}(s)=\int_{0}^{\infty} e_{\lambda}^{-s}(t) f(t) d t,
$$

if the integral converges. Several properties and interesting formulas for the degenerate Laplace transform were derived in [11]. Motivated by this study, in this paper, we have considered degenerate Sumudu transform by the following improper integral:

$$
G_{\lambda}(u)=\mathbf{S}_{\lambda}[f(t)]=\frac{1}{u} \int_{0}^{\infty} e_{\lambda}^{\frac{-1}{u}}(t) f(t) d t, \quad u \in\left(-\tau_{1}, \tau_{2}\right) \text { and } \lambda \in(0, \infty) .
$$

for

$$
f(t) \in A_{\lambda}=\left\{f(t) \mid \exists M, \tau_{1}, \tau_{2}>0 \text { such that }|f(t)|<M e_{\lambda}^{1 / \tau_{j}}(|t|), \text { if } t \in(-1)^{j} \times[0, \infty)\right\} .
$$

We then have provided some of their properties and relations. We have also derived degenerate Sumudu transforms of power functions, degenerate sine, degenerate cosine, degenerate hyperbolic sine, degenerate hyperbolic cosine, degenerate exponential function, and function derivatives. We have attained a relation between degenerate Sumudu transform and degenerate gamma function. Moreover, we have investigated a scale preserving theorem for the degenerate Sumudu transform. Lastly, we have shown that the degenerate Sumudu transform is the theoretical dual transform to the degenerate Laplace transform.

\section{Declaration of Competing Interest}

The author declare that he has no known competing financial interests or personal relationships that could have appeared to influence the work reported in this paper. 


\section{References}

[1] Asiru, M. U. Further properties of the Sumudu transform and its applications, Int. J. Math. Edu. Sci. Tech. 33(3), 2002, 44-449.

[2] F. B. M. Belgacem, A. A. Karaballi, and S. L. Kalla, Analytical investigations of the Sumudu transform and applications to integral production equations, Math. Prob. Eng. 2003(3), 2003, 103-118.

[3] Belgacem, F. B. M. Introducing and analysing deeper Sumudu properties. Nonlinear Studies, 13(1), 2006, $23-41$.

[4] Belgacem, F. B. M., Karaballi, A. A. Sumudu transform fundamental properties investigations and applications, J. Appl. Math. Stoc. Anal. Article ID 91083, 2016, 1-23.

[5] Carlitz, L. Degenerate Stirling, Bernoulli and Eulerian numbers. Utilitas Math. 1975, 15, 51-88.

[6] Carlitz, L. A degenerate Staudt-Clausen theorem. Arch Math (Basel). 1956, 7, 28-33.

[7] Debnath, L. Integral Transforms and Their Applications, CRC Press, Florida, 1995.

[8] Duran, U.; Sadjang, P.N. On Gould-Hopper-based fully degenerate poly-Bernoulli polynomials with a $q$-parameter, Mathematics, 2019, 7, 121.

[9] Kim. T.; Kim, D.S. Degenerate Bernstein polynomials. RASCAM. 2018, doi: 10.1007/s13398-018-0594-9.

[10] Kim, D.S.; Kim, T.; Mansour, T.; Seo, J.-J. Degenerate Mittag-Leffler polynomials. Appl. Math. Comput. 2016, 274, $258-266$

[11] Kim. T.; Kim, D.S. Degenerate Laplace transform and degenerate gamma function, Russ. J. Math. Phys. 2017, 24, 241-248.

[12] Kim, Y.; Kim, B.M.; Jang, L.-C.; Kwon, J. A Note on modified degenerate gamma and Laplace transformation. Symmetry $2018,10,471$.

[13] Kim, T., Kim, D.S. Note on the degenerate gamma function. Russ. J. Math. Phys. 27, 352-358 (2020). https://doi.org/10.1134/S1061920820030061.

[14] Kim, D.S.; Kim, T., Lee, H. A note on Degenerate Euler and Bernoulli polynomials of complex variable, Symmetry, 2019, $11,1168$.

[15] Lim, D. Some identities of degenerate Genocchi polynomials. Bull. Korean Math. Soc. 2016, 53, 569-579.

[16] Poularikas A. D. The Transforms and Applications Handbook, The Electrical Engineering Handbook Series, CRC Press, Florida, 1996.

[17] Saadeh, R.; Qazza, A.; Burqan, A. A New Integral Transform: ARA Transform and Its Properties and Applications. Symmetry 2020, 12, 925 .

[18] Saif, M., Khan, F., Nisar, K. S., Araci, S., Modified Laplace transform and its properties, J. Math. Comput. SCI-JM, 21 (2020), no. 2, 127-135.

[19] Watugula, G.K. Sumudu transform: A new integral transform to solve differential equations and control engineering problems. Int. J. Math. Edu. Sci. Technol. 1993, 24, 35-43.

[20] Watugala, G. K. The Sumudu transform for functions of two variables, Mathematical Engineering in Industry 8(4), (2002), 293-302.

[21] Weerakoon, S. Application of Sumudu transform to partial differential equations, Int. J. Math. Edu. Sci. Tech., 25(2), 1994, 277-283, DOI: 10.1080/0020739940250214. 